\title{
Paramétrisation de la contrainte de cisaillement en interaction houle-courant par le modèle de la turbulence $\boldsymbol{k}$ - $\omega$
}

\author{
Hassan SMAOUI ${ }^{1}$, Abdellatif OUAHSINE ${ }^{2}$, Achraf Mahdi FARHANE ${ }^{3}$
}

1. CETMEF-LHN, Laboratoire Roberval, UMR-CNRS 6253, B.P. 20529 , 60206 Compiègne Cedex, France.

Hassan.Smaoui@developpement-durable.gouv.fr

2. UTC-LHN, Laboratoire Roberval, UMR-CNRS 6253, B.P. 20529, 60206 Compiègne Cedex, France.

3. ENSAM-Meknès, Dpt Mécanique des fluides, B.P. 4024, Beni M'Hamed, 50000 Meknès, Maroc.

\section{Résumé :}

Dans ce travail, nous nous proposons d'appliquer le modèle $\mathrm{k}-\omega$ à un écoulement combinant houle et courant afin de proposer une nouvelle paramétrisation des contraintes de cisaillement (maximale et moyenne) de cet écoulement. La précision d'une telle paramétrisation est nécessaire pour une bonne estimation des échanges à l'interface fluide-sédiment qui conditionne le transport sédimentaire (charriage et suspension). Une comparaison des performances des modèles classiques de turbulence : k- $\omega$ et k- $\varepsilon$ appliqués à un écoulement purement oscillatoire sera également donnée.

Mots-clés :

Houle-courant - turbulence - modèle k- $\omega$ - modèle k- $\varepsilon$ - contrainte de cisaillement interface fluide-sédiment.

\section{Introduction}

Depuis la fin des années 70, les modèles à deux équations ont été considérés comme un outil efficace et fiable pour la modélisation de la turbulence en écoulements géophysiques. Il existe une grande variété de modèles, cependant seuls deux d'entre eux sont fréquemment utilisés: le premier modèle k-kL est celui proposé par MELLOR et YAMADA (1982), ( $\mathrm{k}$ est l'énergie cinétique turbulente ECT et L est la longueur de mélange). Le second est le modèle k- $\varepsilon$ proposé par RODI (1987).

Parmi les modèles à deux équations rarement appliqués aux écoulements géophysiques, citons, par exemple, le modèle k- $\omega$ proposé par WILCOX (1998) qui résout deux équations de transport (la première pour l'ECT $\mathrm{k}$ et la seconde pour le taux de dissipation spécifique $\omega$ ). Un des avantages du modèle k- $\omega$ démontré par WILCOX est sa capacité à reproduire correctement les caractéristiques d'un écoulement dans la couche visqueuse près des parois solides sans recours à un traitement spécial qu'exige le modèle k- $\varepsilon$ (k- $\varepsilon$ à faible nombre de Reynolds). Notons également que le modèle k- $\omega$ est 
plus performant pour des écoulements présentant une renverse des gradients de pression.

Dans ce travail, dans un premier temps, nous présentons une comparaison des performances des modèles de la turbulence k- $\omega$ et k- $\varepsilon$ appliqués à un écoulement purement oscillatoire. Dans un second temps, nous appliquons le modèle k- $\omega$ pour proposer une nouvelle paramétrisation de la contrainte de cisaillement d'un écoulement combinant une houle et un courant.

\section{Présentation du modèle}

Le problème à étudier est celui de la couche limite que nous considérons unidirectionnelle. Dans ce cas, l'équation de la conservation de la quantité du mouvement se réduit à :

$$
\frac{\partial \mathrm{u}}{\partial \mathrm{t}}=-\frac{1}{\rho} \frac{\partial \mathrm{p}}{\partial \mathrm{x}}+\frac{\partial}{\partial \mathrm{z}}\left(\left(v_{\mathrm{m}}+v_{\mathrm{t}}\right) \frac{\partial \mathrm{u}}{\partial \mathrm{z}}\right)
$$

Où $\mathrm{u}$ est la composante horizontale de la vitesse, $\mathrm{p}$ est la pression, $\rho$ est la masse volumique de l'eau, $v_{\mathrm{m}}$ est la viscosité moléculaire et $v_{\mathrm{t}}$ est la viscosité turbulente.

L'écoulement au-dessus de la couche limite est, au départ une houle progressive dont la composante horizontale de vitesse $\mathrm{U}_{\mathrm{h}}$ est déterminée, selon la théorie linéarisée des ondes de gravité en eau peu profonde par :

$\mathrm{U}_{\mathrm{h}}=\hat{\mathrm{U}}_{\mathrm{h}} \sin \left(\omega_{\mathrm{h}} \mathrm{t}-\mathrm{k}_{\mathrm{h}} \mathrm{x}\right)$

où $\hat{U}_{\mathrm{h}}$ est l'amplitude de la vitesse, $\omega_{\mathrm{h}}$ la pulsation et $\mathrm{k}_{\mathrm{h}}$ est le nombre d'onde de la houle. $\mathrm{Si}$, en plus, la houle satisfait la condition $\hat{\mathrm{U}}_{\mathrm{h}} / \mathrm{C}_{\mathrm{h}}<<1$ (où $\mathrm{C}_{\mathrm{h}}$ est la célérité de la houle), HUTNH-THANH (1990) montre que l'équation (1) se ramène à :

$$
\frac{\partial \mathrm{u}}{\partial \mathrm{t}}=\frac{\partial \mathrm{U}_{\mathrm{h}}}{\partial \mathrm{t}}+\frac{\partial}{\partial \mathrm{z}}\left(\left(v_{\mathrm{m}}+\mathrm{v}_{\mathrm{t}}\right) \frac{\partial \mathrm{u}}{\partial \mathrm{z}}\right)
$$

Pour fermer le système, $v_{t}$ peut être exprimée à partir de divers modèles de la turbulence, comme par exemple les modèles k-L, k- $\varepsilon$ ou k- $\omega$ et bien d'autres.

Dans ce travail, nous comparons dans un premier temps, les performances des modèles $\mathrm{k}-\varepsilon$ et $\mathrm{k}-\omega$ sur un écoulement de type couche limite généré par la propagation d'une onde purement sinusoïdale.

\subsection{Le modèle k- $\omega$}

Pour le modèle $\mathrm{k}-\omega$, la viscosité turbulente $v_{\mathrm{t}}$ est en fonction de l'énergie cinétique turbulente $\mathrm{k}$ et de sa dissipation spécifique $\omega$ :

$v_{\mathrm{t}}=\gamma^{*} \frac{\mathrm{k}}{\omega}$

où la dissipation spécifique de l'ECT est définie par $\omega=\varepsilon /\left(\beta^{*} \mathrm{k}\right)$, où $\gamma^{*}$ et $\beta^{*}$ sont des constantes empiriques (tableau 1). 
Les équations de transport du modèle k- $\omega$ pour l'évolution de l'ECT et de sa dissipation spécifique $\omega$ sont données par WILCOX (1998) :

$$
\begin{aligned}
& \frac{\partial \mathrm{k}}{\partial \mathrm{t}}=v_{\mathrm{t}}\left(\frac{\partial \mathrm{u}}{\partial \mathrm{z}}\right)^{2}+\frac{\partial}{\partial \mathrm{z}}\left(\left(v_{\mathrm{m}}+\frac{v_{\mathrm{t}}}{\sigma^{*}}\right) \frac{\partial \mathrm{k}}{\partial \mathrm{z}}\right)-\beta^{*} \mathrm{k} \omega \\
& \frac{\partial \omega}{\partial \mathrm{t}}=\alpha \frac{\omega}{\mathrm{k}} v_{\mathrm{t}}\left(\frac{\partial \mathrm{u}}{\partial \mathrm{z}}\right)^{2}+\frac{\partial}{\partial \mathrm{z}}\left(\left(v_{\mathrm{m}}+\frac{v_{\mathrm{t}}}{\sigma^{*}}\right) \frac{\partial \omega}{\partial \mathrm{z}}\right)-\beta \omega^{2}
\end{aligned}
$$

Tableau 1. Constantes empiriques du modèle $k-\omega$.

\begin{tabular}{lllll}
\hline$\sigma^{*}$ & $\beta^{*}$ & $\gamma^{*}$ & $\alpha$ & $\beta$ \\
\hline 2,00 & 0,09 & 1,00 & 0,55 & 0,075 \\
\hline
\end{tabular}

\subsection{Le modèle k- $\varepsilon$}

Pour ce modèle, la viscosité turbulente $v_{t}$ s'obtient en remplaçant dans (3) la dissipation spécifique $\omega$ par l'expression $\varepsilon /\left(\beta^{*} \mathrm{k}\right)$ :

$v_{\mathrm{t}}=\mathrm{C}_{\mu} \frac{\mathrm{k}^{2}}{\varepsilon}$

Les équations de transport du modèle k- $\varepsilon$ pour l'évolution de l'ECT et de sa dissipation $\varepsilon$ sont données par RODI (1987) :

$$
\begin{aligned}
& \frac{\partial \mathrm{k}}{\partial \mathrm{t}}=v_{\mathrm{t}}\left(\frac{\partial \mathrm{u}}{\partial \mathrm{z}}\right)^{2}+\frac{\partial}{\partial \mathrm{z}}\left(\left(v_{\mathrm{m}}+\frac{v_{\mathrm{t}}}{\sigma_{\mathrm{k}}}\right) \frac{\partial \mathrm{k}}{\partial \mathrm{z}}\right)-\varepsilon \\
& \frac{\partial \varepsilon}{\partial \mathrm{t}}=\frac{\varepsilon}{\mathrm{k}} \mathrm{C}_{\varepsilon} v_{\mathrm{t}}\left(\frac{\partial \mathrm{u}}{\partial \mathrm{z}}\right)^{2}+\frac{\partial}{\partial \mathrm{z}}\left(\left(v_{\mathrm{m}}+\frac{v_{\mathrm{t}}}{\sigma_{\varepsilon}}\right) \frac{\partial \varepsilon}{\partial \mathrm{z}}\right)-\frac{\varepsilon}{\mathrm{k}} \mathrm{C}_{\varepsilon 2} \varepsilon
\end{aligned}
$$

où $C_{\mu}, \sigma_{\varepsilon}, \sigma_{\mathrm{k}}, \mathrm{C}_{\varepsilon 1}, \mathrm{C}_{\varepsilon 2}$ sont des constantes empiriques (tableau 2).

Tableau 2. Constantes empiriques du modèle $k-\varepsilon$.

\begin{tabular}{lllll}
\hline$C_{\mu}$ & $\sigma_{\mathrm{k}}$ & $\sigma_{\varepsilon}$ & $\mathrm{C}_{\varepsilon 1}$ & $\mathrm{C}_{\varepsilon 2}$ \\
\hline 0,09 & 1,00 & 1,30 & 1,44 & 1,92 \\
\hline
\end{tabular}

\subsection{Conditions aux limites et initiales}

La résolution des équations (2-8) nécessite la donnée des conditions aux limites de la colonne d'eau (au fond et à la surface).

$\mathrm{Au}$ fond situé à $\mathrm{z}=\mathrm{z}_{0}=\mathrm{k}_{\mathrm{n}} / 30 \quad\left(\mathrm{k}_{\mathrm{n}}\right.$ est la rugosité équivalente de Nikuradse) on considère la condition de non glissement pour la vitesse $u$. Si l'on suppose pour les deux modèles l'équilibre entre la production et la dissipation dans la couche logarithmique, on dérivera une condition de type Dirichlet pour l'ECT (k), sa dissipation $\varepsilon$ et sa dissipation spécifique $\omega$ (tableau 3). 
A la surface, située au niveau $\mathrm{z}=\mathrm{Z}_{\mathrm{h}}$, on impose la condition de symétrie (écoulement potentiel). Notons que la valeur de $\mathrm{z}_{\mathrm{h}}$ doit être choisie à un niveau où l'influence de la turbulence est tout à fait négligeable. En général, on prend un niveau qui est supposé plus grand que l'épaisseur de la couche limite.

Tableau 3. Conditions aux limites pour les modèles $k-\omega$ et $k-\varepsilon$.

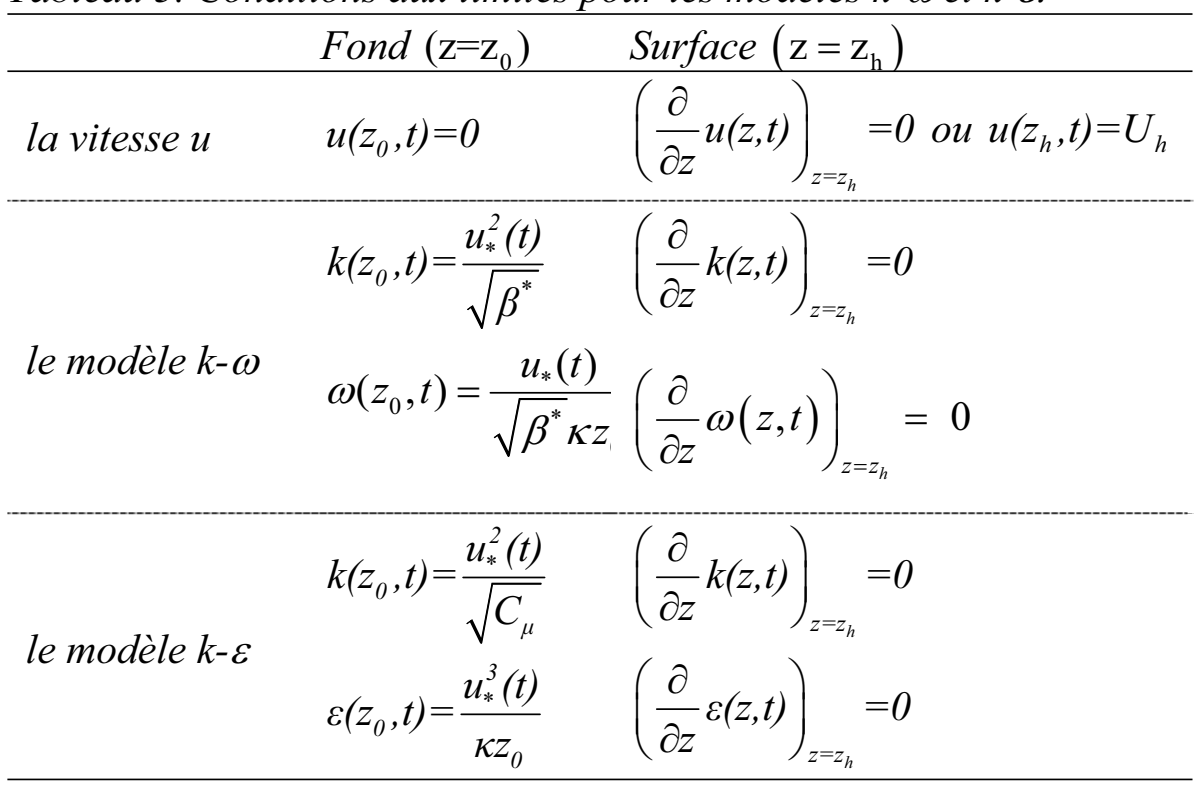

Comme condition initiale, le modèle démarre d'un écoulement permanent de vitesse $U_{c}$ avant de lui superposer une houle régulière. Les variables turbulentes $(\mathrm{k}, \varepsilon, \omega)$ ont été initialisées à des valeurs de l'ordre de $10^{-6}$ afin d'éviter des singularités dans le système d'équations à résoudre.

\section{Résolution numérique}

Pour bien reproduire par le modèle les fortes variations au voisinage de la paroi, nous avons utilisé un maillage en trois couches (couche de fond, intermédiaire et de surface) dans lequel la couche de fond a été maillée de façon très fine (de l'ordre du millimètre). Pour limiter les procédures d'interpolation au cours des calculs, la vitesse $u$ et les variables turbulentes $\left(\mathrm{k}, \omega, \varepsilon, v_{\mathrm{t}}\right)$ ont été calculées sur deux grilles décalées par le schéma centré.

L'ensemble des équations (1-8) avec leurs conditions aux limites a été discrétisé par la méthode des différences finies en espace et en temps. Les dérivées partielles spatiales ont été approchées par un schéma centré. Le schéma d'Euler implicite a été adopté pour les dérivées temporelles de l'ensemble des équations du modèle. Notons que la discrétisation des équations a abouti à la résolution d'un système linéaire tri-diagonal. Une procédure de relaxation a été nécessaire pour éviter des instabilités numériques dues aux termes non linéaires dans les équations $(4,5$ et 7,8$)$. 


\section{Comparaison des modèles $k-\omega$ et $k-\varepsilon$}

Plusieurs études expérimentales existent sur la couche limite de fond due à un mouvement purement oscillatoire (JONSSON, 1963; JONSSON \& CARLSON, 1976; SUMER et al., 1986 ; SLEATH, 1987). Les résultats de ces études constituent une base de données largement utilisée pour la validation des modèles numériques.

En ce qui nous concerne, nous comparons succinctement les résultats des modèles k- $\omega$ et k- $\varepsilon$ aux mesures effectuées par JONSSON (1963). Le tableau 4 résume les principaux paramètres de ces mesures ( $\hat{a}_{h}=\hat{U}_{h} T / 2 \pi$ est l'amplitude orbitale de la houle).

Sur la figure 1 sont présentés les profils des vitesses mesurées et simulées par les modèles $\mathrm{k}-\omega$ et $\mathrm{k}-\varepsilon$ durant la phase d'accélération de la houle. On peut constater globalement que les profils calculés par les deux modèles sont en bon accord avec ceux mesurés. Cependant, nous précisons que le modèle $\mathrm{k}-\omega$ prédit mieux l'écoulement dans la couche près de la paroi (par exemple pour les phases $60^{\circ}$ et $300^{\circ}$ ). Au delà de cette couche, les deux modèles sont parfaitement équivalents.

Tableau 4. Paramètres expérimentaux.

\begin{tabular}{llllll}
\hline$\hat{U}_{h}(\mathrm{~cm} / \mathrm{s})$ & $T(\mathrm{~s})$ & $\hat{a}_{h}(\mathrm{~cm})$ & $z_{h}(\mathrm{~cm})$ & $k_{n}(\mathrm{~cm})$ & $\hat{a}_{h} / k_{n}$ \\
\hline 211,0 & 8,4 & 282,23 & 100,0 & 2,27 & 124,0 \\
\hline
\end{tabular}
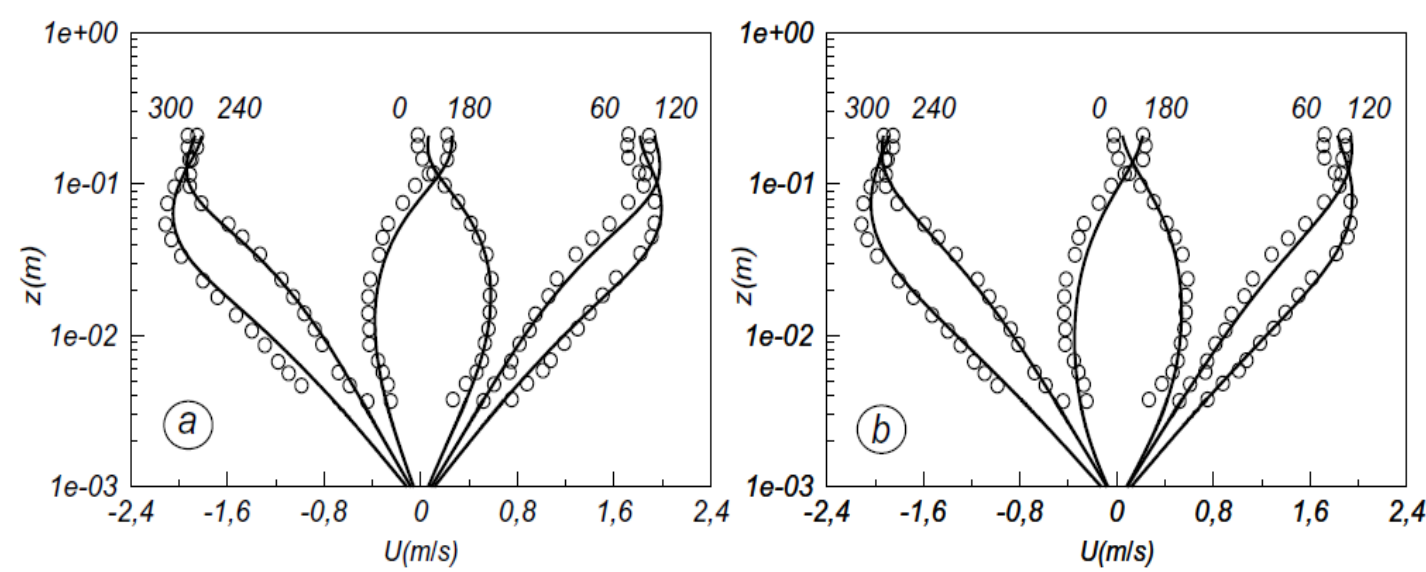

Figure 1. (a) Mesures et modèle $k$ - $\varepsilon$. (b) Mesures et modèle $k$ - $\omega$. (cercle $=$ mesures, trait $=$ modèle) .

\section{Paramétrisation de $\mathbf{f}_{\mathrm{h}}, \tau_{\max }$ et $\tau_{\mathrm{m}}$.}

Dans cette partie, nous considérons le cas d'une houle en présence d'un courant stationnaire $U_{c}$. En présence d'un tel courant la pression totale est la somme d'une pression due à la houle $\mathrm{p}_{\mathrm{h}}$ et d'une pression due au courant $\mathrm{p}_{\mathrm{c}}\left(\mathrm{p}=\mathrm{p}_{\mathrm{h}}+\mathrm{p}_{\mathrm{c}}\right)$. D'après DAVIS et al., (1988), dans la couche limite, les gradients de pression peuvent être considérés indépendants de z. Par conséquent, cette approximation permet de reformuler l'équation de la quantité de mouvement (1) sous la forme: 


$$
\frac{\partial \mathrm{u}}{\partial \mathrm{t}}=\hat{\mathrm{U}}_{\mathrm{h}} \omega_{\mathrm{h}} \cos \left(\omega_{\mathrm{h}} \mathrm{t}-\phi\right)+\frac{\tau_{\mathrm{c}}}{\mathrm{h}}+\frac{\partial}{\partial \mathrm{z}}\left(\left(v_{\mathrm{m}}+v_{\mathrm{t}}\right) \frac{\partial \mathrm{u}}{\partial \mathrm{z}}\right)
$$

\subsection{Paramétrisation du coefficient du frottement $f_{h}$}

Pour un écoulement généré par la houle, plusieurs expressions (SWART, 1974 ; JONSSON, 1966; MYRHAUG, 1989) du coefficient de frottement théoriques et empiriques $\mathrm{f}_{\mathrm{h}}$ ont été proposées sous la forme :

$\mathrm{f}_{\mathrm{h}}=\mathrm{c}_{1} \operatorname{Exp}\left[\mathrm{c}_{2}\left(\frac{\hat{\mathrm{a}}_{\mathrm{h}}}{\mathrm{k}_{\mathrm{n}}}\right)^{\mathrm{c}_{3}}\right] \quad \mathrm{c}_{1}, \mathrm{c}_{2}, \mathrm{c}_{3}$ sont des constantes réelles à déterminer.

La contrainte de cisaillement $\hat{\tau}_{\mathrm{h}}$ due à la houle est estimée alors par : $\hat{\tau}_{\mathrm{h}} / \rho=0.5 \mathrm{f}_{\mathrm{h}} \hat{\mathrm{U}}_{\mathrm{h}}^{2}$. En faisant varier $\hat{a}_{h} / k_{n}$ de 1 à $10^{4}$ et par application successive du modèle k- $\omega$ pour calculer $\hat{\tau}_{\mathrm{h}}$, nous en déduisons une série de valeurs de $\mathrm{f}_{\mathrm{h}}$. L'application de la méthode des moindres carrés non linéaires à ces valeurs nous a permis d'obtenir, avec une bonne précision, les constantes $\mathrm{c}_{1}, \mathrm{c}_{2}, \mathrm{c}_{3}$ résumées dans le tableau 5 .

Notons que le coefficient de frottement calculé par nos constantes est très proche de ceux obtenus par voie expérimentale (SLEATH, 1987; SUMMER et al., 1987 ; SIMONS et al., 1988).

Tableau 5. Coefficients d'ajustement de l'expression (10).

\begin{tabular}{lll}
\hline$c_{1}$ & $c_{2}$ & $c_{3}$ \\
\hline $1,24 \times 10^{-3}$ & 4,12 & $-0,12$ \\
\hline
\end{tabular}

5.2 Paramétrisation des contraintes de cisaillement (maximale et moyenne)

Plusieurs paramétrisations de $\tau_{\max }$ (contrainte maximale) et $\tau_{\mathrm{m}}$ (contrainte de moyenne sur une période) basées sur des modèles de turbulences à zéro-équation ont été proposées dans la littérature (figure 2). Nous proposons, ici une nouvelle paramétrisation de $\tau_{\max }$ et $\tau_{\mathrm{m}}$ en s'appuyant sur le modèle k- $\omega$. Cette paramétrisation requière trois paramètres d'entrée: la rugosité relative $\mathrm{z}_{0} / \mathrm{h}$, l'excursion relative $\hat{a}_{h} / k_{n}$, et l'angle entre la houle et le courant $\phi_{\mathrm{hc}}$.

La paramétrisation de $\tau_{\max }$ et $\tau_{\mathrm{m}}$ consiste à chercher "les meilleures" expressions analytiques approchant les quantités $\mathrm{Y}=\tau_{\max } /\left(\tau_{\mathrm{c}}+\hat{\tau}_{\mathrm{h}}\right)$ et $\mathrm{y}=\tau_{\mathrm{m}} /\left(\tau_{\mathrm{c}}+\hat{\tau}_{\mathrm{h}}\right)$ en fonction de la quantité $\mathrm{x}=\tau_{\mathrm{c}} /\left(\tau_{\mathrm{c}}+\hat{\tau}_{\mathrm{h}}\right)$, (SOULSBY et al., 1993).

Pour situer nos résultats par rapport à ceux présentés dans le cadre du programme Européen MAST-G8-M (figure 2), nous considérons les mêmes expressions de x, Y et y adoptés par les différents participants au programme.

$$
\mathrm{Y}=1+\mathrm{ax}^{\mathrm{m}}(1-\mathrm{x})^{\mathrm{n}} \quad ; \quad \mathrm{y}=\mathrm{x}\left[1+\mathrm{bx}^{\mathrm{p}}(1-\mathrm{x})^{\mathrm{q}}\right]
$$

Où toutes les constantes $(\mathrm{a}, \mathrm{b}, \mathrm{m}, \mathrm{n}, \mathrm{p}, \mathrm{q})$ que l'on note par $\varphi$, sont sous la forme : 
$\varphi=\left(\varphi_{1}+\varphi_{2} \mid \cos \left(\left.\phi_{\mathrm{hc}}\right|^{\mathrm{I}}\right)+\left(\varphi_{3}+\varphi_{4} \mid \cos \left(\left.\phi_{\mathrm{hc}}\right|^{\mathrm{J}}\right) \log _{10}\left(\mathrm{f}_{\mathrm{h}} / \mathrm{f}_{\mathrm{c}}\right)\right.\right.$

La paramétrisation de $\left(\tau_{\max }\right)$ et $\left(\tau_{\mathrm{m}}\right)$ revient donc à chercher les constantes $\varphi_{1}, \varphi_{2}, \varphi_{3}, \varphi_{4}, \mathrm{I}, \mathrm{J}$ qui minimisent l'écart entre les résultats du modèle et les expressions (11). Après application d'une procédure d'optimisation non linéaire sous contraintes, nous obtenons les coefficients $\varphi_{1}, \varphi_{2}, \varphi_{3}, \varphi_{4}, \mathrm{I}, \mathrm{J}$. Ainsi, nous déterminons les expressions (11) issues de la modélisation de la turbulence par k- $\omega$.

La figure 2, présente la paramétrisation de $\mathrm{Y}$ et y pour $\phi_{h c}=0, z_{0} / h=10^{-4}$ et $\hat{a}_{h} / z_{0}=10^{4}$. On observe que notre paramétrisation de $\mathrm{Y}$ est proche de celles proposées par DAVIS et al. (1988) et FREDSOE (1984) qui sont les plus utilisées dans la littérature. En revanche, la figure 2.b montre que la paramétrisation de y est très proche de celle proposée par GRANT et MADSEN (1979).
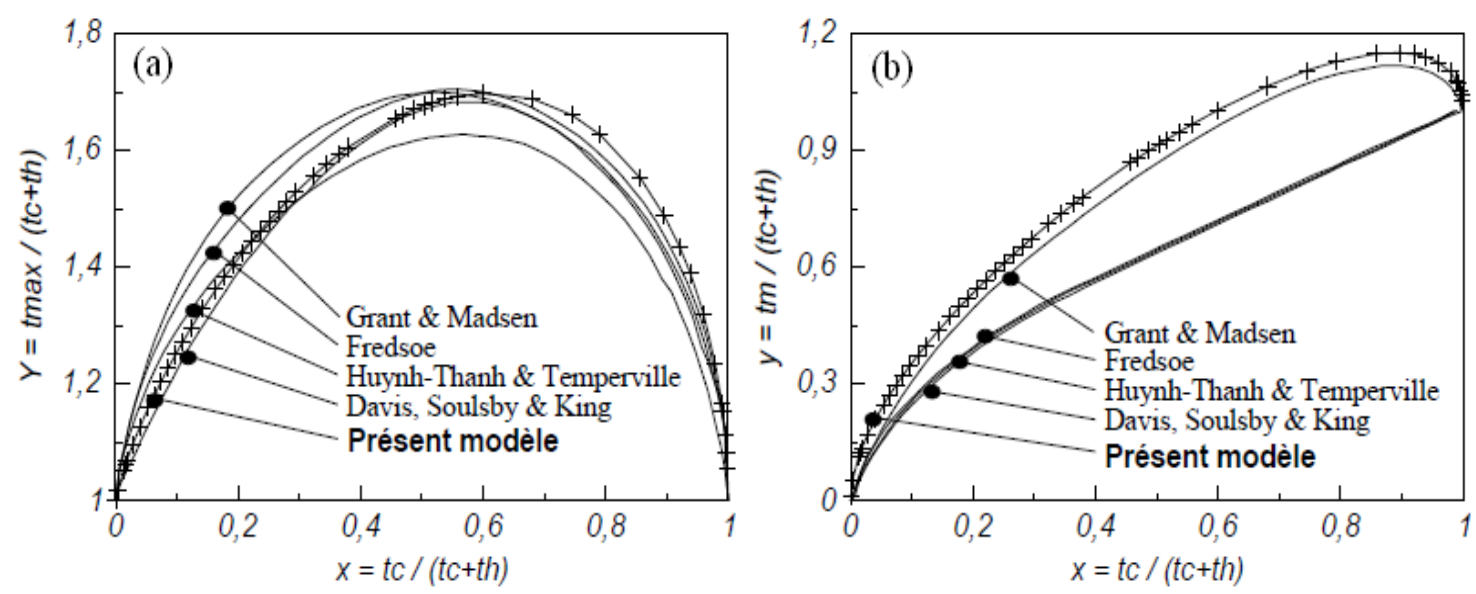

Figure 2. Paramétrisation de $\tau_{\max }$ et $\tau_{\mathrm{m}}$ par le modèle $k$ - $\omega$.

\section{Conclusion}

La couche limite turbulente oscillatoire sur un fond rugueux plat a été étudiée grâce aux modèles de fermeture turbulente $\mathrm{k}-\omega$ et $\mathrm{k}-\varepsilon$. Il ressort de cette étude que le modèle $\mathrm{k}-\omega$ est capable de reproduire correctement les caractéristiques d'un écoulement dans la couche visqueuse prés des parois solides sans recours à un traitement spécial, qu'exige le modèle k- $\varepsilon$. Basé sur ce constat, le modèle $\mathrm{k}-\omega$ a été appliqué pour proposer une nouvelle expression algébrique du coefficient de frottement $\mathrm{f}_{\mathrm{h}}$ dû à la propagation d'une houle. Les valeurs obtenues sont très proches de celles obtenues par d'autres expressions couramment utilisées dans la littérature.

Le modèle $\mathrm{k}-\omega$ a été appliqué également pour proposer une nouvelle paramétrisation des contraintes de cisaillement $\tau_{\max }$ et $\tau_{\mathrm{m}}$ en interaction houle-courant. Bien que le modèle $k-\omega$ nécessite encore des validations en interaction houle-courant, notre paramétrisation est très proche de certaines paramétrisations largement utilisées par la communauté scientifique. 
Thème 1 - Hydrodynamique côtière

\section{Références bibliographiques}

FREDSOE J. (1984). Turbulent boundary layer in wave-current motion. Journal of Hydraulics Engineering, ASCE 110, pp 1103-1120. doi:10.1061/(ASCE)0733-9429(1984)110:8(1103)

DAVIS A.G., SOULSBY R.L., KING H.L. (1988). A numerical model of the combined wave and current bottom boundary layer. Journal of Geophysical Research, 93(C1), pp 491-508. doi:10.1029/JC093iC01p00491

GRANT W.D., MADSEN O.S. (1979). Combined wave and current interaction with rough bottom. Journal of Geophysical Research, 84(C4), pp 1797-1808. doi:10.1029/JC084iC04p01797

JONSSON I.G. (1963). Measurements in the turbulent wave boundary layer. In Proc 10th congress IAHR, London, U K, pp 85-92.

JONSSON I.G. (1966). Wave boundary layers and friction factors. Proc.10th Int. Conf. on Coastal Engineering, Tokyo, Japan, ASCE, pp 127-148.

JONSSON I.G., CARLSON N.A. (1976). Experimental and theoretical investigations in an oscillatory turbulent boundary layer. J. of Hydraulics. Research, 14 (1), pp 45-60. MELLOR G.L., YAMADA T. (1982). Development of a turbulence closure model for geophysical fluid problems. Review in Geophysical and. Space Physics, 20 (4), pp 851-875. doi:10.1029/RG020i004p00851

MYRHAUG D. (1989). A rational approach to wave friction coefficients for rough, smooth and transitional turbulent flow. Coastal Engineering, 13(1), pp 11-21. doi:10.1016/0378-3839(90)90027-T

RODI W. (1987). Development of a turbulence closure model for geophysical fluid problems. Journal of Geophysical Research 92 (C5), pp 5305-5328. doi:10.1029/JC092iC05p05305

SIMONS R.R., KYRIACOU A., SOULSBY R.L., DAVIS A.G. (1988). Predicting the near bed turbulent flow in waves and currents. In Proc IAHR Symp on Mathematical Modeling of Sediment Transport in the Coastal Zone, Copenhagen, Denmark, pp 33-37. SLEATH J.F.A. (1987). Turbulent oscillatory flow over rough beds. Journal of Fluid Mechanics. 182, pp 369-409. doi:10.1017//50022112087002374

SOULSBY R.L., HAMM L., KLOPMAN G., MYRHAUG D., SIMONS R.R., THOMAS G.P. (1993). Wave-current interaction within and outside the bottom boundary layer. Coastal Engineering, 21, pp 41-69. doi:10.1016/0378-3839(93)90045-A

SUMER B.M., JENSEN B.L., FREDSOE J. (1987). Turbulence in oscillatory boundary layers. In C.Comte-Bellot and J. Mathieu (Editors), Advances in Turbulence, SpringerVerlag, Berlin, pp 556-567.

SWART D.H. (1974). Offshore sediment transport and equilibrium beach profiles. Delft Hydraulics Lab. Publ. 131, Delft, The Netherlands.

WILCOX D.C. (1998). Turbulence modeling for CFD. DCW Industry Incorporation, Canada, Calif. 